\title{
Determination of Effective Atomic Numbers Using Different Methods for Some Low- $Z$ Materials
}

\author{
Vishwanath P. Singh, ${ }^{1,2}$ N. M. Badiger, ${ }^{1}$ and Nil Kucuk ${ }^{3}$ \\ ${ }^{1}$ Department of Physics, Karnatak University, Dharwad, Karnatak 580003, India \\ ${ }^{2}$ Health Physics Section, Kaiga Atomic Power Station 3 and 4, NPCIL, Karwar 581400, India \\ ${ }^{3}$ Department of Physics, Faculty of Arts and Sciences, Uludag University, 16059 Bursa, Turkey \\ Correspondence should be addressed to Vishwanath P. Singh; kudphyvps@rediffmail.com
}

Received 18 April 2014; Revised 4 July 2014; Accepted 9 July 2014; Published 7 August 2014

Academic Editor: Hasan Mahmood Khan

Copyright (C) 2014 Vishwanath P. Singh et al. This is an open access article distributed under the Creative Commons Attribution License, which permits unrestricted use, distribution, and reproduction in any medium, provided the original work is properly cited.

In the present work, different methods were used to determine the effective atomic numbers of some low- $Z$ materials, namely, polyethylene (PE), polystyrene (PS), polypropylene (PP), Perspex (PX), polycarbonate (PC), nylon 6-6 (PA-6), plaster of Paris (POP), and TH/L2. These methods are the direct method, the interpolation method, Auto- $Z_{\text {eff }}$ software, and single value XMuDat computer program. Some of the results obtained were compared with experimental data wherever possible. It can be concluded from this work that the effective atomic numbers calculated with the direct, the interpolation and Auto- $Z_{\text {eff }}$ methods demonstrate a good agreement in Compton scattering and pair production energy regions. A large difference in the effective atomic numbers calculated by the direct and the interpolation methods of low- $Z$ materials was also observed in photoelectric and pair production regions. It was determined that PE, PS, PX, and PA- 6 were equivalent to adipose and muscle; POP was equivalent to cortical bone; TH/L2 was equivalent to thyroid tissue; PP was equivalent to yellow bone marrow and adipose tissues; PC was equivalent to spongiosa.

\section{Introduction}

Simulation of radiation dose distribution in human organs and tissues is possible by tissue equivalent materials. ICRU report 44 [1] describes various types of tissue substitutes for human organs and tissues. Tissue substitutes are being used for phantom, medical applications, radiology, nuclear engineering, health physics, radiation physics, radiation dosimetry, radiation protection, and space research. The effective atomic number is photon interaction parameter which is used for dosimetric properties. The effective atomic numbers can be calculated using different methods such as the direct method, the interpolation method, Auto- $Z_{\text {eff }}$ software, and single value XMuDat computer program. Many researchers have made extensive effective atomic numbers studies on a variety of materials such as gaseous mixtures [2], dosimetric materials [3-5], alloys [6-9], semiconductors $[10,11]$, building materials $[12]$, glasses $[13,14]$, soils $[15,16]$, amino acids [17], fatty acids [18], minerals [19], and biological samples $[20,21]$.
In this study, the effective atomic numbers for low- $Z$ materials have been determined using the direct, the interpolation, Auto- $Z_{\text {eff }}$, and XMuDat methods. The theoretical results were compared with experimental data wherever possible. This study should be useful for readily available effective atomic numbers of the low- $Z$ materials for choice of appropriate computational method.

\section{Calculation Methods}

Mass attenuation coefficient and attenuation cross-section data are available in photon energy range of $1 \mathrm{keV}$ to $100 \mathrm{GeV}$ in the form XCOM program [22] which has been transformed to windows operating system software WinXCom [23]. The atomic number and atomic masses of the elements have been taken from recent report on atomic weight of elements 2011, IUPAC [24]. The effective atomic numbers are derived by calculation of the mass attenuation coefficients and atomic cross-sections of the elements of compound/mixture. The elemental compositions of the low $Z$ materials used in this 
TABLE 1: Elemental compositions of the low- $Z$ materials.

\begin{tabular}{|c|c|c|c|c|c|c|c|c|c|c|}
\hline \multirow{2}{*}{ Tissue substitute } & \multicolumn{10}{|c|}{ Element weight (\%) } \\
\hline & $\mathrm{H}$ & $\mathrm{C}$ & $\mathrm{N}$ & $\mathrm{O}$ & $\mathrm{Na}$ & $\mathrm{P}$ & S & $\mathrm{Cl}$ & $\mathrm{K}$ & $\mathrm{Ca}$ \\
\hline Polyethylene (PE) & 14.37 & 85.63 & - & - & - & - & - & - & - & - \\
\hline Polystyrene (PS) & 11.18 & 88.82 & - & - & - & - & - & - & - & - \\
\hline Polypropylene (PP) & 14.37 & 85.63 & - & - & - & - & - & - & - & - \\
\hline Perspex (PX) & 8.050 & 59.98 & - & 31.96 & - & - & - & - & - & - \\
\hline Polycarbonate (PC) & 7.060 & 78.92 & - & 14.02 & - & - & - & - & - & - \\
\hline nylon 6-6 (PA-6) & 9.800 & 63.68 & 12.38 & 14.14 & - & - & - & - & - & - \\
\hline Plaster of Paris (POP) & 2.300 & - & - & 55.80 & - & - & 18.6 & - & - & 23.3 \\
\hline TH/L2 & 10.00 & 13.60 & 2.200 & 73.50 & 0.2 & 0.1 & - & 0.1 & 0.2 & - \\
\hline
\end{tabular}

study are given in Table 1 . The calculation methods for the effective atomic numbers of the low- $Z$ materials are described in the following subsections.

2.1. Auto- $Z_{\text {eff }}$ Method. Auto- $Z_{\text {eff }}$ method is user-friendly software in visual basic for rapid computation of the average atomic numbers and spectral-weighted mean atomic numbers. The Auto- $Z_{\text {eff }}$ surpasses dubious power-law approach. In this method, effective atomic number is determined via exploitation of the smooth correlation between atomic crosssection and atomic number. A matrix of cross-sections was constructed spanning atomic number $Z=1-100$ for photon energies ranging between $10 \mathrm{keV}$ and $1 \mathrm{GeV}$ and the cross-sections of polyelemental media are calculated by linear additivity. The cross-sectional values are constructed with the cross-section matrix as a function of $Z$, and an effective atomic number is obtained by the interpolation of $Z$ values between adjacent cross-section data [25].

2.2. Direct Method. Calculation of the effective atomic numbers $Z_{\text {eff,PI }}$ of the low- $Z$ materials for total photon interaction was carried out by using practical formula [26]. The formula is given below:

$$
Z_{\mathrm{eff}, \mathrm{PI}}=\frac{\sum_{i} f_{i} A_{i}(\mu / \rho)_{i}}{\sum_{j}\left(f_{j} A_{j} / Z_{j}\right)(\mu / \rho)_{j}}
$$

where $f_{i}$ is molar fraction in the mixture/compound, $\mu$ is linear attenuation coefficient, $\rho$ is density, $\mu / \rho$ is mass attenuation coefficient, $A$ is atomic weight, $Z$ is atomic number, and the ratio, $A / Z$, between the atomic mass and the atomic number is approximately constant.

2.3. Interpolation Method. Mass attenuation coefficient values $\mu_{m}(=\mu / \rho)$ are derived for the selected low $Z$ materials using the mixture rule: $(\mu / \rho)_{\text {material }}=\sum_{i}^{n} w_{i}(\mu / \rho)_{i}$, where $w_{i}$ is the proportion by weight and $(\mu / \rho)_{i}$ is mass attenuation coefficient of the $i$ th element tabulated in XCOM [22] software or WinXCom [23] software. The quantity $w_{i}$ is given by $w_{i}=n_{i} A_{i} / \sum_{j}^{n} n_{j} A_{j}$ with condition $\sum_{i}^{n} w_{i}=1$, where $A_{i}$ is the atomic weight of the $i$ th element and $n_{i}$ is the number of formula units in the compounds.
The attenuation cross-section $(\sigma)$ values of the composite material are computed by using the following relation:

$$
\sigma=\frac{\left(\mu_{m}\right)}{N \sum_{i}\left(w_{i} / A_{i}\right)}\left(\frac{\text { barn }}{\text { molecule }}\right) \text {, }
$$

where $N=6.023 \times 10^{23}$ is Avogadro's number in atom g ${ }^{-1}, w_{i}$ is weight fraction of the $i$ th element in a molecule of the tissue substitutes, and $A_{i}$ is the atomic weight of the $i$ th element in a molecule. $w_{i}$ and $A_{i}$ are both dimensionless quantities.

The equivalent atomic numbers using the logarithmic interpolation formula is given as follows:

$$
Z_{\mathrm{eq}}=\frac{Z_{1}\left(\log \sigma_{2}-\log \sigma\right)+Z_{2}\left(\log \sigma-\log \sigma_{1}\right)}{\log \sigma_{2}-\log \sigma_{1}},
$$

where $\sigma_{1}$ and $\sigma_{2}$ are elemental atomic cross-section (barn/atom) for atomic numbers of elements corresponding to $Z_{1}$ and $Z_{2}$, and $\sigma$ is atomic cross-section of the composite materials lying between the $\sigma_{1}$ and $\sigma_{2}$.

2.4. XMuDat Method. XMuDat computer program is able to produce a single value effective atomic number for compounds [27]. The XMuDat uses the following formula for calculation of the effective atomic number:

$$
Z_{\mathrm{eff}, \mathrm{XMuDat}}=\left(\sum_{i} \alpha_{i} Z_{i}^{m-1}\right)^{1 /(m-1)}
$$

where $\alpha_{i}$ is the fractional number of the electrons of the $i$ th element and $m$ is a constant between 3 and 5. It is preferred that $m$ is set to 3.6 for materials with $Z_{\text {eff }}<6$ and 4.1 for materials with $Z_{\text {eff }}>6$ [28].

\section{Results and Discussion}

The variation of the effective atomic numbers of the low$Z$ materials with photon energy is shown in Figures 1(a), 1(b), 1(c), 1(d), 1(e), 1(f), 1(g), and 1(h). The effective atomic numbers below $10 \mathrm{keV}$ were not compared due to large uncertainty $( \pm 25 \%)$ in Auto- $Z_{\text {eff }}[25]$. From Figures 1(a), $1(\mathrm{~b}), 1(\mathrm{c}), 1(\mathrm{~d}), 1(\mathrm{e}), 1(\mathrm{f}), 1(\mathrm{~g})$, and $1(\mathrm{~h})$ it is clearly seen that the effective atomic numbers calculated by Auto- $Z_{\text {eff }}$, the direct, and the interpolation methods are in very good 
TABLE 2: (a) Effective atomic numbers calculated with the direct and the interpolation methods for PE, PS, and PP with experimental data. (b) Effective atomic numbers calculated with the direct and the interpolation methods for PX, PC, and PA-6 with experimental data.

(a)

\begin{tabular}{|c|c|c|c|c|c|c|c|c|c|}
\hline \multirow{2}{*}{ Energy $(\mathrm{MeV})$} & \multicolumn{3}{|c|}{$\mathrm{PE}$} & \multicolumn{3}{|c|}{ PS } & \multicolumn{3}{|c|}{ PP } \\
\hline & $Z_{\text {eff,PI }}$ & $Z_{\mathrm{eq}}$ & $\mathrm{a}$ & $Z_{\text {eff,PI }}$ & $Z_{\mathrm{eq}}$ & $\mathrm{b}$ & $Z_{\text {eff,PI }}$ & $Z_{\mathrm{eq}}$ & $\mathrm{b}$ \\
\hline 0.013 & 4.688 & 4.462 & 4.5 & 4.947 & 4.702 & - & 4.688 & 4.460 & - \\
\hline 0.017 & 4.027 & 4.191 & 4.1 & 4.359 & 4.479 & - & 4.027 & 4.188 & - \\
\hline 0.022 & 3.515 & 3.813 & 3.8 & 3.872 & 4.163 & - & 3.515 & 3.810 & - \\
\hline 0.026 & 3.234 & 3.504 & - & 3.593 & 3.877 & - & 3.234 & 3.500 & - \\
\hline 0.032 & 3.017 & 3.205 & 3.1 & 3.371 & 3.594 & - & 3.017 & 3.201 & - \\
\hline 0.033 & 2.988 & 3.160 & - & 3.340 & 3.551 & - & 2.988 & 3.155 & - \\
\hline 0.044 & 2.823 & 2.913 & 2.9 & 3.167 & 3.284 & - & 2.823 & 2.910 & - \\
\hline 0.054 & 2.762 & 2.833 & - & 3.102 & 3.175 & - & 2.762 & 2.829 & - \\
\hline 0.060 & 2.742 & 2.806 & - & 3.081 & 3.138 & 2.98 & 2.742 & 2.803 & 2.64 \\
\hline 0.081 & 2.703 & 2.756 & - & 3.040 & 3.067 & - & 2.704 & 2.753 & - \\
\hline 0.123 & 2.681 & 2.727 & - & 3.016 & 3.025 & - & 2.681 & 2.723 & - \\
\hline 0.279 & 2.670 & 2.713 & - & 3.004 & 3.005 & - & 2.670 & 2.709 & - \\
\hline 0.356 & 2.669 & 2.712 & - & 3.002 & 3.003 & - & 2.669 & 2.708 & - \\
\hline 0.511 & 2.668 & 2.711 & - & 3.001 & 3.002 & 3.00 & 2.668 & 2.707 & 2.67 \\
\hline 0.662 & 2.667 & 2.710 & - & 3.001 & 3.001 & 3.00 & 2.667 & 2.707 & 2.66 \\
\hline 1.115 & 2.667 & 2.711 & - & 3.001 & 3.002 & - & 2.667 & 2.708 & - \\
\hline 1.173 & 2.667 & 2.710 & - & 3.001 & 3.001 & 3.00 & 2.667 & 2.707 & 2.66 \\
\hline 1.274 & 2.667 & 2.710 & - & 3.001 & 3.000 & 3.00 & 2.667 & 2.706 & 2.66 \\
\hline 1.333 & 2.668 & 2.710 & - & 3.001 & 3.001 & 3.00 & 2.668 & 2.706 & 2.66 \\
\hline
\end{tabular}

Values have been reported by Parthasaradhi et al. [29]. ${ }^{\mathrm{b}}$ Values have been reported by Kucuk et al. [30].

(b)

\begin{tabular}{|c|c|c|c|c|c|c|c|c|c|c|c|c|}
\hline \multirow{2}{*}{ Energy $(\mathrm{MeV})$} & \multicolumn{6}{|c|}{$\mathrm{PX}$} & \multicolumn{3}{|c|}{$\mathrm{PC}$} & \multicolumn{3}{|c|}{ PA-6 } \\
\hline & $Z_{\text {eff,PI }}$ & $Z_{\mathrm{eq}}$ & $\mathrm{c}$ & $\mathrm{d}$ & $\mathrm{a}$ & $\mathrm{e}$ & $Z_{\text {eff,PI }}$ & $Z_{\mathrm{eq}}$ & $\mathrm{a}$ & $Z_{\text {eff,PI }}$ & $Z_{\mathrm{eq}}$ & $\mathrm{c}$ \\
\hline 0.013 & 6.210 & 5.606 & - & - & 5.6 & - & 5.789 & 5.374 & 5.8 & 5.707 & 5.221 & - \\
\hline 0.017 & 5.615 & 5.453 & - & - & 5.4 & - & 5.281 & 5.226 & 5.5 & 5.072 & 5.035 & - \\
\hline 0.022 & 5.014 & 5.201 & - & - & 5.2 & - & 4.788 & 4.996 & 5.3 & 4.483 & 4.738 & - \\
\hline 0.026 & 4.612 & 4.931 & - & 4.822 & & - & 4.469 & 4.757 & - & 4.115 & 4.442 & - \\
\hline 0.032 & 4.258 & 4.580 & - & - & 4.6 & - & 4.195 & 4.468 & 4.8 & 3.806 & 4.088 & - \\
\hline 0.033 & 4.206 & 4.518 & - & 4.399 & - & - & 4.156 & 4.418 & - & 3.762 & 4.028 & - \\
\hline 0.044 & 3.903 & 4.088 & - & - & - & - & 3.926 & 4.077 & 4.4 & 3.509 & 3.677 & - \\
\hline 0.054 & 3.785 & 3.906 & - & - & - & 3.53 & 3.837 & 3.935 & - & 3.413 & 3.531 & - \\
\hline 0.060 & 3.745 & 3.848 & - & 3.823 & - & & 3.808 & 3.890 & - & 3.381 & 3.481 & - \\
\hline 0.081 & 3.669 & 3.736 & - & - & - & 3.43 & 3.751 & 3.803 & - & 3.320 & 3.385 & - \\
\hline 0.123 & 3.627 & 3.673 & - & - & - & 3.62 & 3.718 & 3.755 & - & 3.286 & 3.330 & - \\
\hline 0.279 & 3.606 & 3.641 & 3.6 & - & - & & 3.702 & 3.729 & - & 3.268 & 3.303 & 3.1 \\
\hline 0.356 & 3.604 & 3.640 & - & - & - & 3.71 & 3.700 & 3.729 & - & 3.266 & 3.301 & - \\
\hline 0.511 & 3.602 & 3.637 & - & - & - & 3.67 & 3.699 & 3.726 & - & 3.265 & 3.299 & - \\
\hline 0.662 & 3.601 & 3.636 & 3.4 & - & - & 3.52 & 3.698 & 3.726 & - & 3.264 & 3.298 & 2.9 \\
\hline 1.115 & 3.601 & 3.637 & 3.3 & - & - & & 3.698 & 3.727 & - & 3.264 & 3.299 & 2.9 \\
\hline 1.173 & 3.601 & 3.636 & - & - & - & 3.61 & 3.698 & 3.726 & - & 3.264 & 3.298 & - \\
\hline 1.274 & 3.601 & 3.635 & - & - & - & 3.56 & 3.698 & 3.725 & - & 3.264 & 3.297 & - \\
\hline 1.333 & 3.601 & 3.635 & - & - & - & 3.57 & 3.698 & 3.725 & - & 3.264 & 3.297 & - \\
\hline
\end{tabular}

aalues have been reported by Parthasaradhi et al. [29]. ${ }^{\mathrm{c}}$ Values have been reported by Kumar et al. [31]. ${ }^{\mathrm{d}}$ Values have been reported by Vijayakumar et al. [32]. ${ }^{\mathrm{e}}$ Values have been reported by El-Kateb and Abdul-Hamid [33]. 


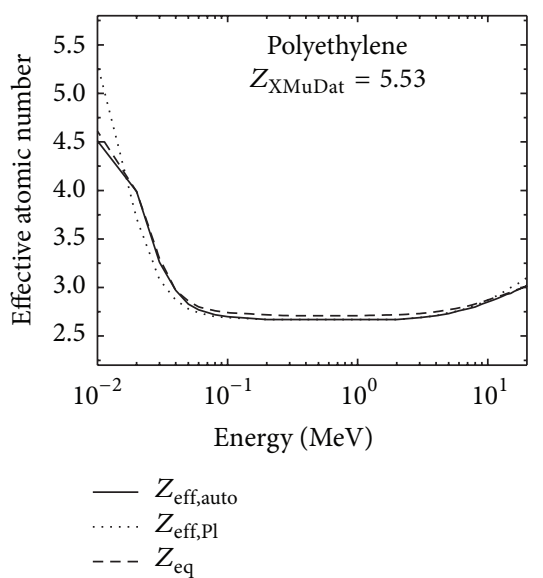

(a)

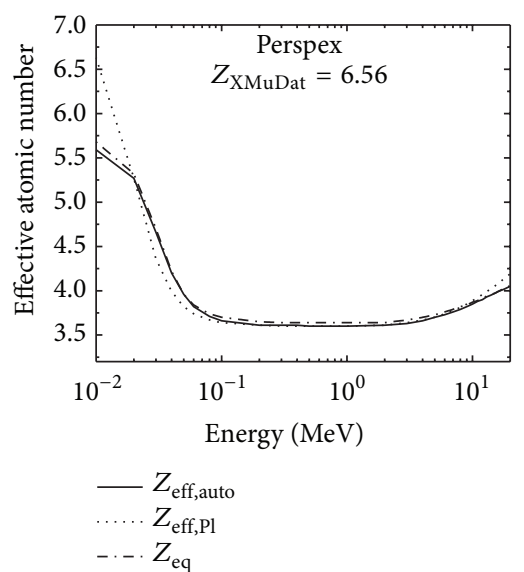

(d)

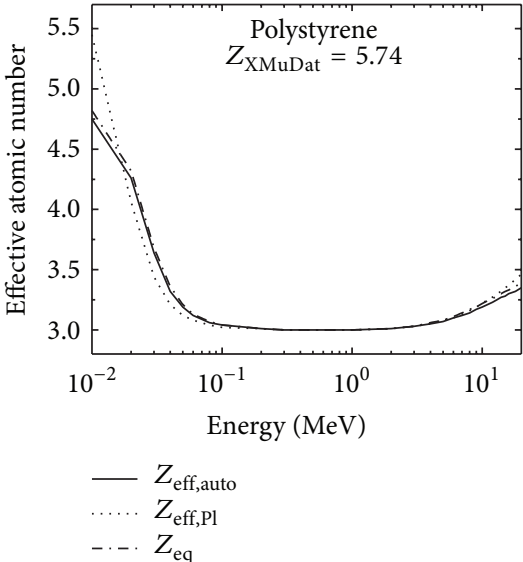

(b)
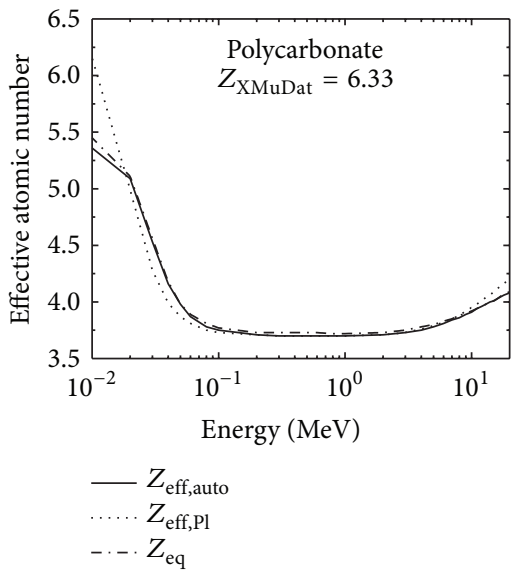

(e)

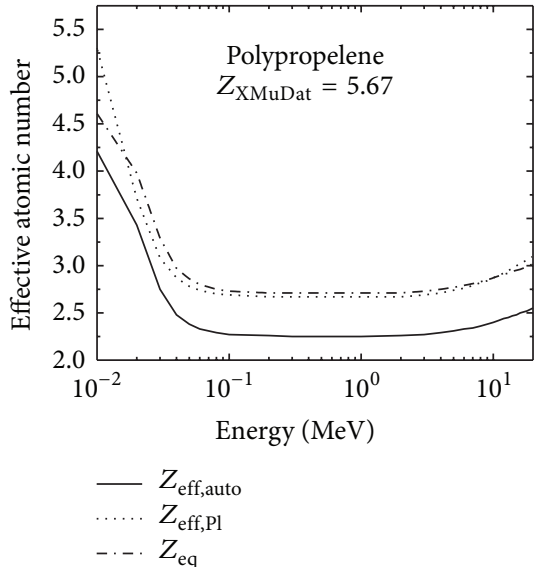

(c)

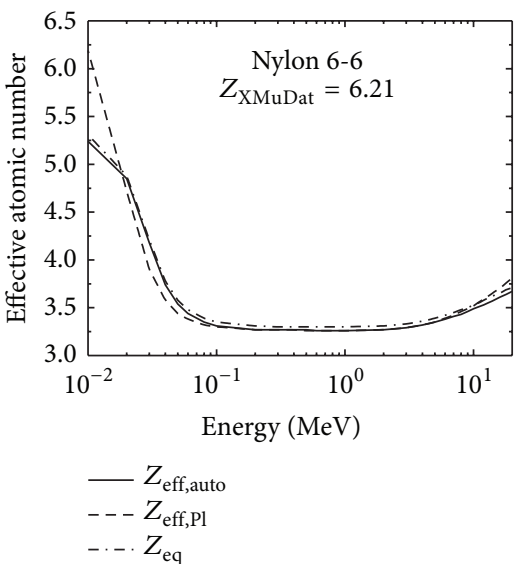

(f)

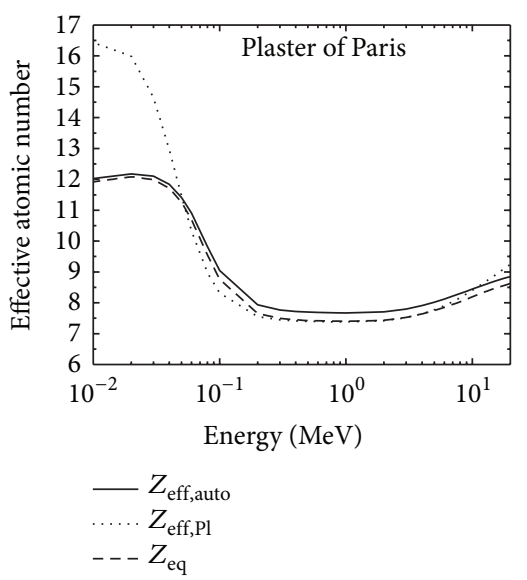

(g)

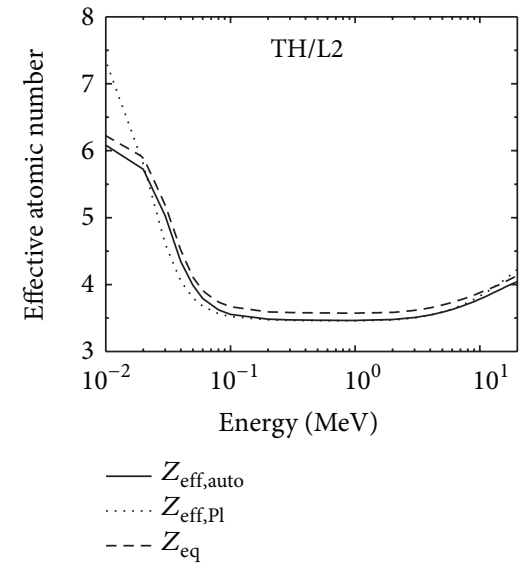

(h)

FIgURE 1: The effective atomic numbers of low- $Z$ materials as a function of photon energy.

agreement in the energy region $(100 \mathrm{keV} \leq E \leq 20 \mathrm{MeV})$, where the Compton interaction dominates. The effective atomic numbers are constant in the intermediate-energy region, whereas noticeable variation is observed for low$(<20 \mathrm{keV})$ as well as high-energy regions. The effective atomic numbers calculated by the direct method are higher in photoelectric absorption and pair production compared with the interpolation method. The effective atomic numbers calculated by XMuDat method are 5.53, 5.74, 5.67, 6.56, 6.33, and 6.21 for PE, PS, PP, PX, PC, and PA-6, respectively. The effective atomic numbers calculated by Auto- $Z_{\text {eff }}$ for PP were found to be less than the direct and the interpolation methods. The independency of the effective atomic numbers on photon energy in intermediate-energy region observed 


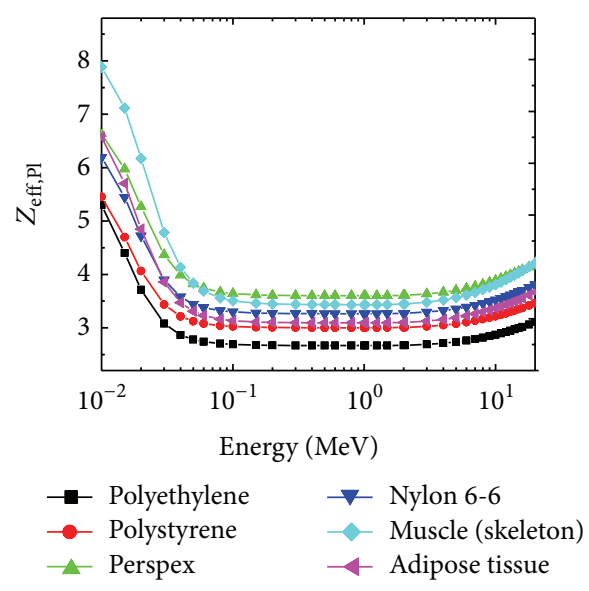

(a)

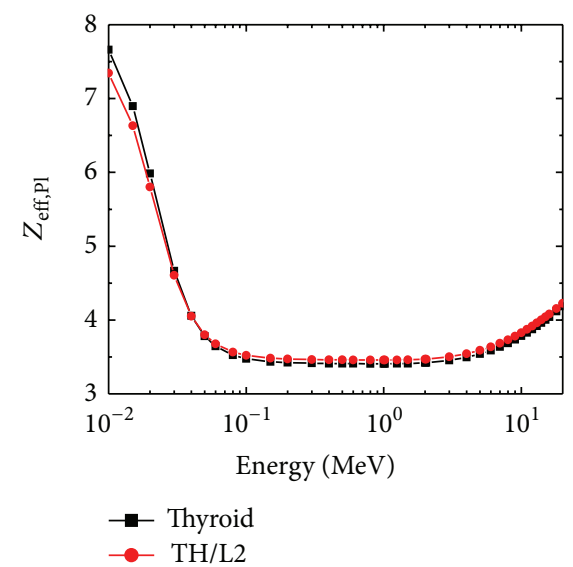

(c)

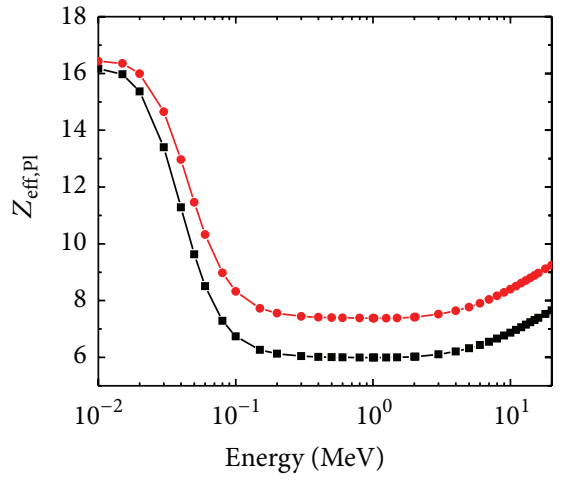

- Cortical bone

- Plaster of Paris

(b)

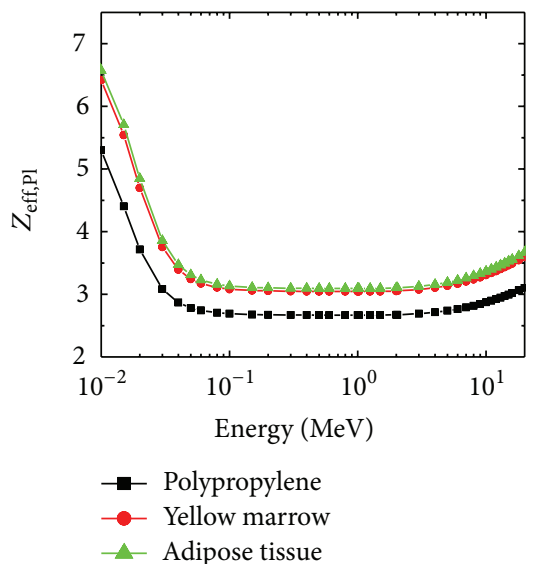

(d)

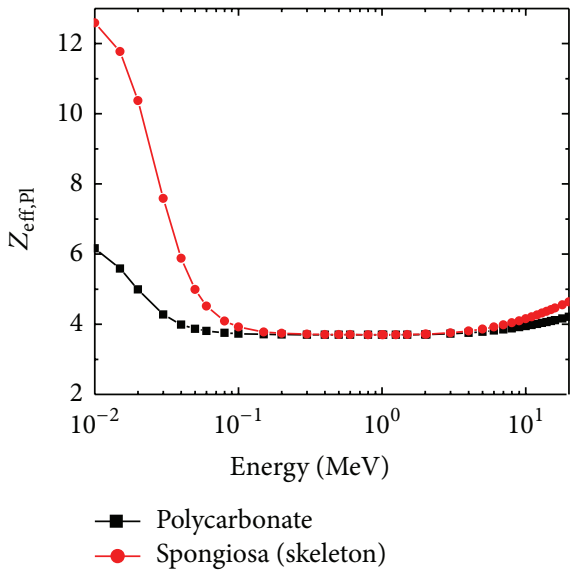

(e)

Figure 2: Comparison of the calculated effective atomic numbers of low- $Z$ materials with human body tissues: (a) muscle (skeleton), (b) cortical bone, (c) thyroid, (d) adipose tissue and yellow bone marrow, and (e) spongiosa (skeleton). 
in our investigation is similar to other various literatures for low- and high- $Z$ elemental composites; however photoelectric absorption and pair production region need further experimental explanation [5]. Also large differences are observed in photoelectric absorption region, which is due to dependency on atomic number of the elements and photon energy. In Compton scattering region, it is found that the effective atomic numbers calculated by all three methods were the same order. The uncertainties in the effective atomic numbers computed by Auto- $Z_{\text {eff }}$ method are of order of $1-2 \%$ for high photon energies.

Comparison of the theoretical effective atomic numbers with experimental data in the literature for PE, PS, and PP is given in Table 2(a). Also comparison of the theoretical effective atomic numbers with experimental data in the literature for PX, PC, and PA-6 is given in Table 2(b). From the tables, it has been clearly seen that the experimental values of the effective atomic numbers were consistent with the theoretical values.

\section{Tissue Substitute and Human Body Tissues}

The calculated $Z_{\text {eff,PI }}$ values of the low- $Z$ materials were compared with the human body tissues in energy range $10 \mathrm{keV}$ to $20 \mathrm{MeV}$ as shown in Figures 2(a), 2(b), 2(c), 2(d), and 2(e). Various types of tissue substitutes for human organs and tissues are muscle (skeleton), cortical bone, thyroid, adipose tissue, yellow bone marrow, and spongiosa (skeleton). The calculated $Z_{\text {eff,PI }}$ values of the low- $Z$ materials are found to be in very good agreement with the human body tissues with insignificant difference in the Compton scattering region. The following results were determined.

(a) PE, PS, PX, and PA-6 were found equivalent to adipose and muscle (skeleton). The $Z_{\text {eff,PI }}$ values vary in the ranges of 3.45-7.89, 3.10-6.57, 2.67-5.30, 3.00$5.45,3.61-6.63$, and 3.27-6.19 for muscle (skeleton), adipose, PE, PS, PX, and PA-6, respectively.

(b) Plaster of Paris (POP) was found equivalent to cortical bone whose $Z_{\text {eff,PI }}$ values vary in range $7.42-16.43$ for POP and 6.02-16.17 for cortical bone respectively.

(c) TH/L2 was found equivalent to thyroid tissue where the $Z_{\text {eff,PI }}$ values vary in range $3.47-7.34$ for TH/L2 and $3.42-7.66$ for thyroid respectively.

(d) PP was found to be equivalent to yellow bone marrow and adipose tissues. The $Z_{\text {eff,PI }}$ values vary in the ranges of $2.67-5.30,3.05-6.42$, and 3.10-6.57 for polypropylene, yellow bone marrow, and adipose tissues, respectively.

(e) PC was found equivalent to spongiosa (skeleton) and $Z_{\text {eff,PI }}$ values vary in the ranges of 3.71-6.16 for polycarbonate and 3.72-12.59 for spongiosa. The reason for large atomic numbers compared with $\mathrm{PC}$ in low-energy is due to high- $Z$ elements $(\mathrm{Na}, \mathrm{Mg}, \mathrm{P}$, $\mathrm{S}$, and $\mathrm{Ca}$ ) in spongiosa (photoelectric cross-section is dependent on $Z^{4-5}$ ).

\section{Conclusions}

In the present work, the theoretical methods were used to determine the effective atomic numbers of some low$Z$ materials (i.e., PE, PS, PP, PX, PA-6, POP, and TH/L2). The direct, the interpolation, and Auto- $Z_{\text {eff }}$ methods demonstrate a good agreement in the effective atomic numbers in Compton scattering and pair production energy regions. A large difference in the effective atomic numbers calculated by the direct and the interpolation methods was observed in photoelectric and pair production regions. It was determined that PE, PS, PX, and PA-6 were equivalent to adipose and muscle (skeleton); POP was equivalent to cortical bone; TH/L2 was equivalent to thyroid tissue; $\mathrm{PP}$ was equivalent to yellow bone marrow and adipose tissues; PC was equivalent to spongiosa (skeleton).

\section{Conflict of Interests}

The authors declare that there is no conflict of interests regarding the publication of this paper.

\section{References}

[1] International Commission on Radiation Units and Measurements ICRU, "Tissue substitutes in radiation dosimetry and measurement," Tech. Rep. 44, ICRU, Betheda , Md, USA, 1989.

[2] V. P. Singh and N. M. Badiger, "Effective atomic numbers, electron densities, and tissue equivalence of some gases and mixtures for dosimetry of radiation detectors," Nuclear Technology \& Radiation Protection, vol. 27, no. 2, pp. 117-124, 2012.

[3] T. K. Kumar, S. Venkataratnam, and K. V. Reddy, "Effective atomic number studies in clay minerals for total photon interaction in the energy region $10 \mathrm{keV}-10 \mathrm{MeV}$," Radiation Physics and Chemistry, vol. 48, no. 6, pp. 707-710, 1996.

[4] Shivaramu and V. Ramprasath, "Effective atomic numbers for photon energy absorption and energy dependence of some thermoluminescent dosimetric compounds," Nuclear Instruments and Methods in Physics Research B, vol. 168, no. 3, pp. 294-304, 2000.

[5] T. Kiran Kumar and K. Venkata Reddy, "Effective atomic numbers for materials of dosimetric interest," Radiation Physics and Chemistry, vol. 50, no. 6, pp. 545-553, 1997.

[6] I. Han, M. Aygun, L. Demir, and Y. Sahin, "Determination of effective atomic numbers for $3 \mathrm{~d}$ transition metal alloys with a new semi-empirical approach," Annals of Nuclear Energy, vol. 39, no. 1, pp. 56-61, 2012.

[7] M. Kurudirek, M. Büyükyıldız, and Y. Özdemir, "Effective atomic num ber study of various alloys for total photon interaction in the energy region of $1 \mathrm{keV}-100 \mathrm{GeV}$," Nuclear Instruments and Methods in Physics Research A, vol. 613, pp. 251256, 2010.

[8] V. R. K. Murty, "Effective atomic numbers for W/Cu alloy for total photon attenuation," Radiation Physics and Chemistry, vol. 71, no. 3-4, pp. 667-669, 2004.

[9] A. H. El-Kateb, R. A. M. Rizk, and A. M. Abdul-Kader, "Determination of atomic cross-sections and effective atomic numbers for some alloys," Annals of Nuclear Energy, vol. 27, no. 14, pp. 1333-1343, 2000.

[10] A. Çelik, U. Çevik, E. Bacaksiz, and N. Çelik, "Effective atomic numbers and electron densities of $\mathrm{CuGaSe}_{2}$ semiconductor in 
the energy range 6-511 keV,' X-Ray Spectrometry, vol. 37, no. 5, pp. 490-494, 2008.

[11] O. Ïçelli, "Measurement of efffective atomic numbers of holmium doped and undoped layered semiconductors via transmission method around the absorption edge," Nuclear Instruments and Methods in Physics Research A, vol. 600, no. 3, pp. 635-639, 2009.

[12] N. Damla, H. Baltas, A. Celik, E. Kiris, and U. Cevik, "Calculation of radiation attenuation coefficients, effective atomic numbers and electron densities for some building materials," Radiation Protection Dosimetry, vol. 150, no. 4, Article ID ncr432, pp. 541-549, 2012.

[13] J. Kaewkhao and P. Limsuwan, "Mass attenuation coefficients and effective atomic numbers in phosphate glass containing $\mathrm{Bi}_{2} \mathrm{O}_{3}, \mathrm{PbO}$ and $\mathrm{BaO}$ at $662 \mathrm{keV}$," Nuclear Instruments and Methods in Physics Research A: Accelerators, Spectrometers, Detectors and Associated Equipment, vol. 619, no. 1-3, pp. 295297, 2010.

[14] H. Gill, G. Kaur, K. Singh, V. Kumar, and J. Singh, "Study of effective atomic numbers in some glasses and rocks," Radiation Physics and Chemistry, vol. 51, no. 4-6, pp. 671-672, 1998.

[15] G. S. Mudahar and H. S. Sahota, "Effective atomic number studies in different soils for total photon interaction in the energy region 10-5000 keV," Applied Radiation and Isotopes, vol. 39, no. 12, pp. 1251-1254, 1988.

[16] N. Kucuk, Z. Tumsavas, and M. Cakir, "Determining photon energy absorption parameters for different soil samples," Journal of Radiation Research, vol. 54, no. 3, pp. 578-586, 2013.

[17] S. Gowda, S. Krishnaveni, and R. Gowda, "Studies on effective atomic numbers and electron densities in amino acids and sugars in the energy range 30-1333 keV," Nuclear Instruments and Methods in Physics Research B: Beam Interactions with Materials and Atoms, vol. 239, no. 4, pp. 361-369, 2005.

[18] S. R. Manohara, S. M. Hanagodimath, and L. Gerward, "Studies on effective atomic number, electron density and kerma for some fatty acids and carbohydrates," Physics in Medicine and Biology, vol. 53, no. 20, pp. N377-386, 2008.

[19] I. Han, L. Demir, and M. Şahin, "Determination of mass attenuation coefficients, effective atomic and electron numbers for some natural minerals," Radiation Physics and Chemistry, vol. 78, no. 9, pp. 760-764, 2009.

[20] V. Manjunathaguru and T. K. Umesh, "Effective atomic numbers and electron densities of some biologically important compounds containing $\mathrm{H}, \mathrm{C}, \mathrm{N}$ and $\mathrm{O}$ in the energy range 145$1330 \mathrm{keV}$," Journal of Physics B: Atomic, Molecular and Optical Physics, vol. 39, no. 18, article 025, pp. 3969-3981, 2006.

[21] N. Koç and H. Özyol, "Z-dependence of partial and total photon interactions in some biological samples," Radiation Physics and Chemistry, vol. 59, no. 4, pp. 339-345, 2000.

[22] M. J. Berger and J. H. Hubbell, XCOM: Photon Cross Sections Database, NBSIR, 1987.

[23] L. Gerward, N. Guilbert, K. B. Jensen, and H. Levring, "WinXCom: a program for calculating X-ray attenuation coefficients," Radiation Physics and Chemistry, vol. 71, no. 3-4, pp. 653-654, 2004.

[24] M. E. Wieser, N. Holden, T. B. Coplen et al., "Atomic weights of the elements 2011 (IUPAC technical report)," Pure and Applied Chemistry, vol. 85, no. 5, pp. 1047-1078, 2013.

[25] M. L. Taylor, R. L. Smith, F. Dossing, and R. D. Franich, "Robust calculation of effective atomic numbers: the auto-Zeff software," Medical Physics, vol. 39, no. 4, pp. 1769-1778, 2012.
[26] S. R. Manohara, S. M. Hanagodimath, K. S. Thind, and L. Gerward, "On the effective atomic number and electron density: a comprehensive set of formulas for all types of materials and energies above $1 \mathrm{keV}$," Nuclear Instruments and Methods in Physics Research B: Beam Interactions with Materials and Atoms, vol. 266, no. 18, pp. 3906-3912, 2008.

[27] R. Nowotny, "XMuDat: photon attenuation data on PC, Tech. Rep. IAEA-NDS-195, International Atomic Energy Agency, Vienna, Austria, 1998, https://www-nds.iaea.org/publications/ iaea-nds/iaea-nds-0195.htm.

[28] D. F. Jackson and D. J. Hawkes, "X-ray attenuation coefficients of elements and mixtures," Physics Reports, vol. 70, no. 3, pp. 169-233, 1981.

[29] K. Parthasaradhi, A. Esposito, and M. Pelliccioni, "Photon attenuation coefficients in tissue equivalent compounds," Applied Radiation and Isotopes, vol. 43, no. 12, pp. 1481-1484, 1992.

[30] N. Kucuk, M. Cakir, and N. A. Isitman, "Mass attenuation coefficients, effective atomic numbers and effective electron densities for some polymers," Radiation Protection Dosimetry, vol. 153, no. 1, pp. 127-134, 2013.

[31] S. P. Kumar, V. Manjunathaguru, and T. K. Umesh, "Effective atomic numbers of some $\mathrm{H}-, \mathrm{C}-, \mathrm{N}$ - and $\mathrm{O}$-based composite materials derived from differential incoherent scattering crosssections," Pramana, vol. 74, no. 4, pp. 555-562, 2010.

[32] R. Vijayakumar, L. Rajasekaran, and N. Ramamurthy, "Effective atomic numbers for photon energy absorption of some lowZ substances of dosimetric interest," Radiation Physics and Chemistry, vol. 62, no. 5-6, pp. 371-377, 2001.

[33] A. H. El-Kateb and A. S. Abdul-Hamid, "Photon attenuation coefficient study of some materials containing hydrogen, carbon and oxygen," Applied Radiation and Isotopes, vol. 42, no. 3, pp. 303-307, 1991. 

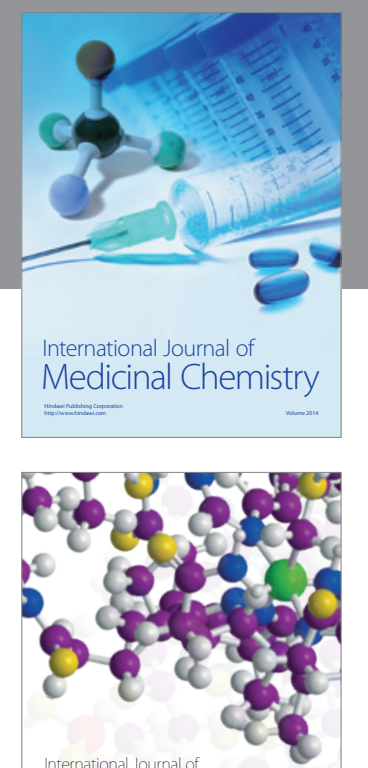

\section{Carbohydrate} Chemistry

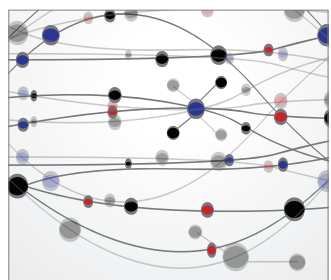

The Scientific World Journal
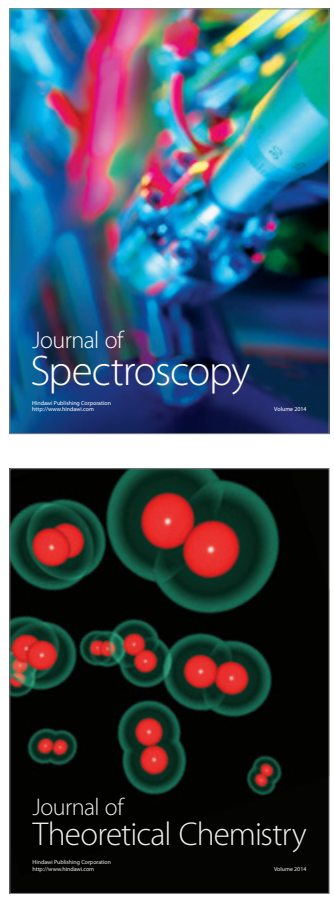
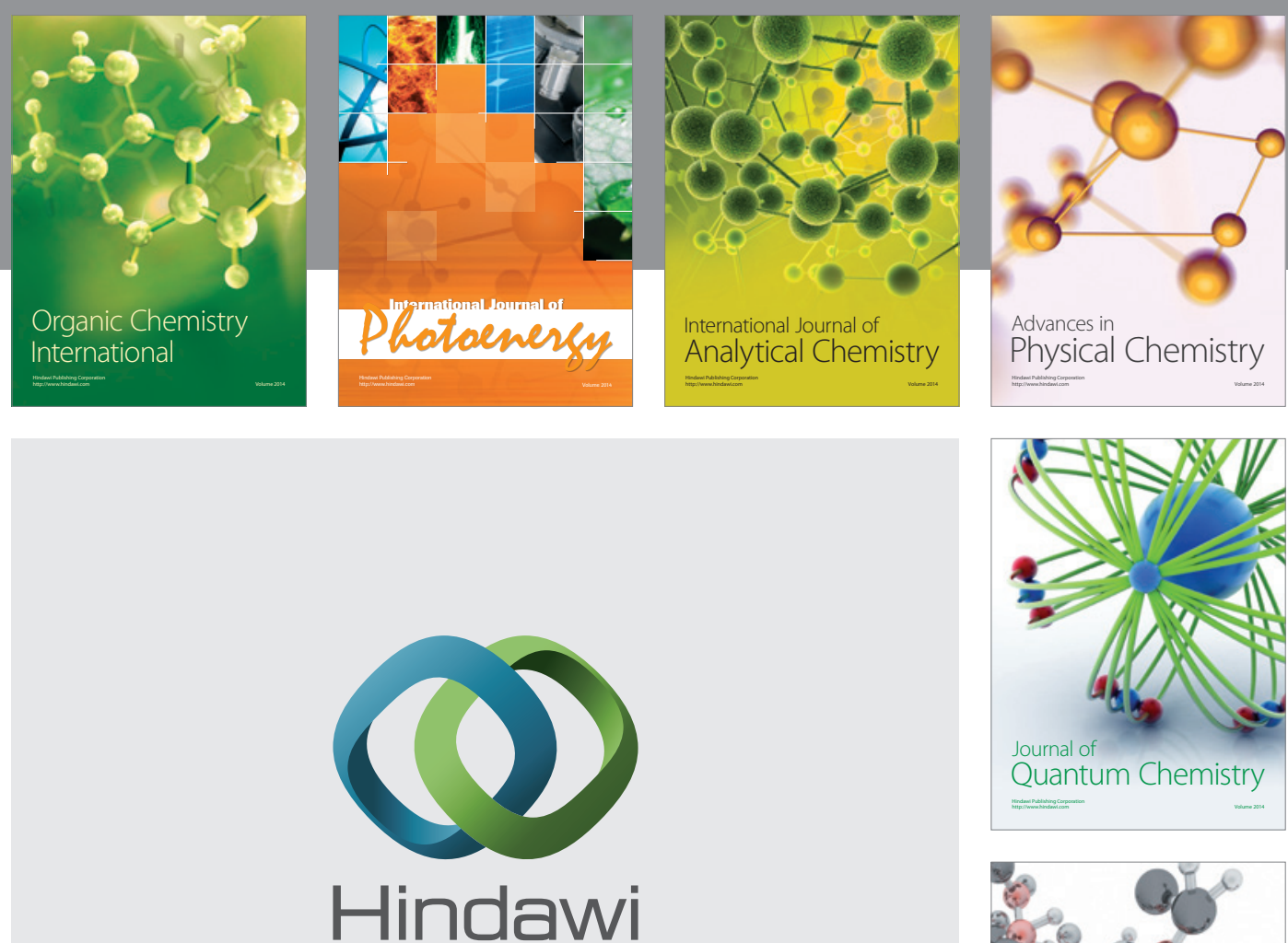

Submit your manuscripts at

http://www.hindawi.com

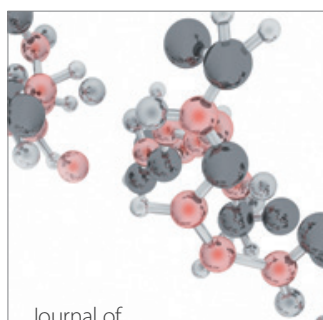

Analytical Methods

in Chemistry

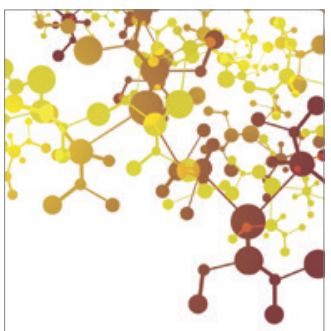

Journal of

Applied Chemistry

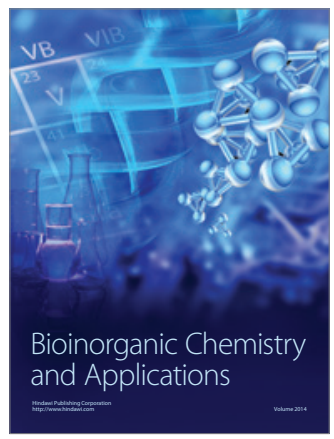

Inorganic Chemistry
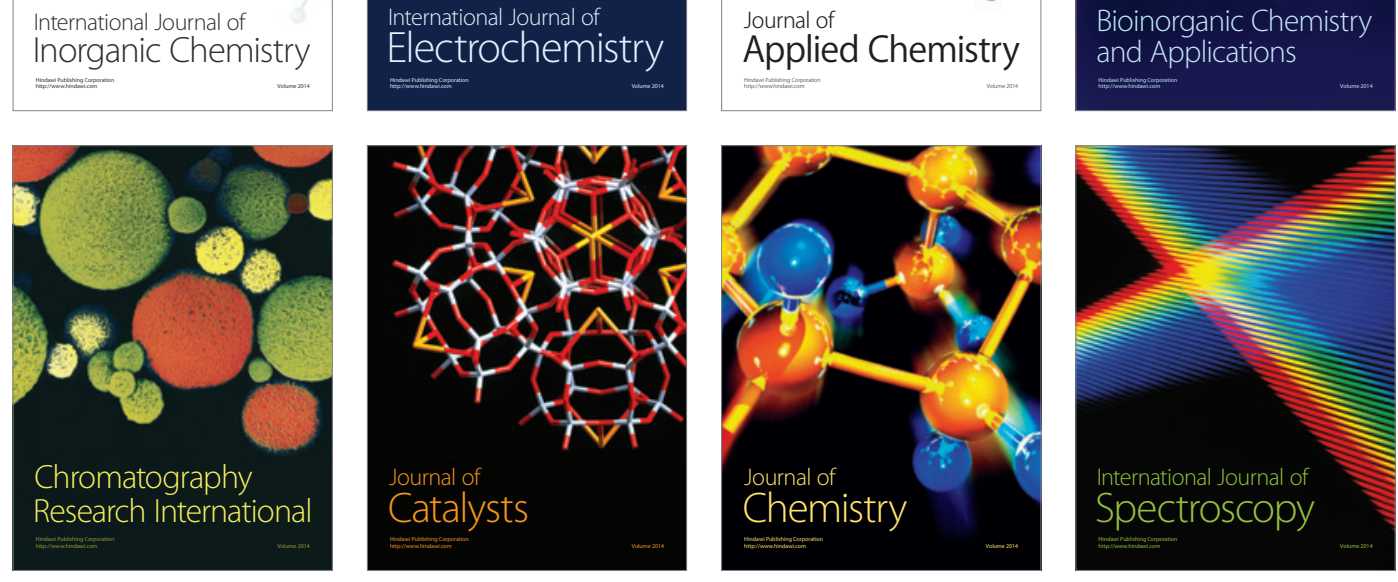